\title{
ON THE EQUIVALENCE OF THE RING, LATTICE, AND SEMIGROUP OF CONTINUOUS FUNCTIONS
}

\author{
MELVIN HENRIKSEN ${ }^{1}$
}

A large number of papers have been published that are devoted to showing that certain algebraic objects obtained by defining operations on the set of all continuous real-valued functions on a suitably restricted topological space determine the space. We mention but a few of them below.

Let $C(X), L(X), S(X)$ denote respectively the ring, lattice, multiplicative semigroup of all continuous real-valued functions on a topological space $X$. Under the assumption that $X$ is a compact Hausdorff space, it was shown in 1937 by M. H. Stone [8] and in 1939 by Gelfand and Kolmogoroff [2] that $C(X)$ determines $X$. In 1947 and 1949, under the same hypothesis, Kaplansky [4] and Milgram [5] showed respectively that each of $L(X), S(X)$ determine $X$. Generalizing these results, Hewitt [3] in 1948 and Shirota [7] in 1952 showed respectively that if $X$ is a $Q$-space (for definition, see [3]), then $C(X)$, respectively, $L(X), S(X)$ determine $X$.

From the point of view of conserving space in the mathematical literature, it is unfortunate that it was not shown first that $L(X)$ or $S(X)$ determine $X$, since any isomorphism between rings of continuous real-valued functions obviously preserves both order and multiplication, so that the corresponding theorems for $C(X)$ would follow. This order of events is not surprising, however, since more is known about the structure of rings than the structure of either lattices or semigroups.

We show below that from the point of view of determining topological spaces, $C(X), L(X)$, and $S(X)$ are equivalent. While this follows easily from Shirota's theorem, we are motivated to publish it by the fact that yet another cycle of the sort described above has begun. In particular, in 1954 and 1955, Pursell [6 [ ${ }^{2}$ and F. W. Anderson [1] have shown respectively that if $X$ is completely regular and its points are $G_{\delta}$-sets, then $C(X), L(X)$ determines $X$.

Presented to the Society, November 26, 1955; received by the editors September $10,1955$.

1 The author was supported by the National Science Foundation, contract No. NSF-G1129.

2 Pursell assumes in addition that $X$ is normal. 
THEOREM. For any topological spaces $X, Y$, the following are equivalent:

(1) $C(X)$ and $C(Y)$ are isomorphic (as rings).

(2) $L(X)$ and $L(Y)$ are isomorphic (as lattices).

(3) $S(X)$ and $S(Y)$ are isomorphic (as semigroups).

Proof. We show that (2) implies (1) and (3), the remaining cases being similar. It is well known that for every topological space $X$, there is a completely regular Hausdorff space $X^{*}$ such that $L(X)(C(X), S(X))$ and $L\left(X^{*}\right)\left(C\left(X^{*}\right), S\left(X^{*}\right)\right)$ are isomorphic. (One obtains $X^{*}$ by first identifying those points which cannot be separated by continuous functions, inducing the functions $f$ of $C(X)$ on $X^{*}$ in the obvious manner, and then giving $X^{*}$ the weakest topology in which these functions are continuous.) Moreover, Hewitt [3] has shown that every completely regular Hausdorff space $X^{*}$ is a dense subspace of a $Q$-space $v X^{*}$ such that every $f \in L\left(X^{*}\right)$ has a unique extension over $v X^{*}$. Thus, (2) implies that $L\left(v X^{*}\right)$ and $L\left(v Y^{*}\right)$ are isomorphic. By the theorem of Shirota cited above, $v X^{*}$ and $v Y^{*}$ are homeomorphic. Hence, $C\left(v X^{*}\right)\left(S\left(v X^{*}\right)\right)$ and $C\left(v Y^{*}\right)\left(S\left(v Y^{*}\right)\right)$ are isomorphic. Reversing the steps described above, we have (1) and (3).

\section{REFERENCES}

1. F. W. Anderson, $A$ lattice characterization of completely regular $G_{\delta}$-spaces, Proc. Amer. Math. Soc. vol. 6 (1955) pp. 757-765.

2. I. Gelfand and A. N. Kolmogoroff, On rings of continuous functions on topological spaces, C.R. (Doklady) Acad. Sci. URSS. vol. 22 (1939) pp. 11-15.

3. E. Hewitt, Rings of real-valued continuous functions I, Trans. Amer. Math. Soc. vol. 64 (1948) pp. 45-99.

4. I. Kaplansky, Lattices of continuous functions, Bull. Amer. Math. Soc. vol. 53 (1947) pp. 617-623.

5. A. N. Milgram, Multiplicative semigroups of continuous functions, Duke Math. J. vol. 16 (1949) pp. 377-383.

6. L. E. Pursell, An algebraic characterization of fixed ideals in certain function rings, Pacific Journal of Mathematics vol. 5 (1955) pp. 963-999.

7. T. Shirota, A generalization of a theorem of I. Kaplansky, Osaka Math. J. vol. 4 (1952) pp. 121-132.

8. M. H. Stone, A pplication of the theory of Boolean rings to general topology, Trans. Amer. Math. Soc. vol. 41 (1937) pp. 375-481.

PuRdue University 